\title{
Efeito do exerćicio físico aeróbio sobre o perfil lipídico de pacientes idosas, portadoras de Diabetes Mellitus tipo 2, atendidas em Unidade Básica de Saúde, Maringá, Estado do Paraná
}

Effect of aerobic physical exercise on the lipid profile in type 2 diabetic elderly women attended in a basic health unit in Maringá, Paraná State, Brazil

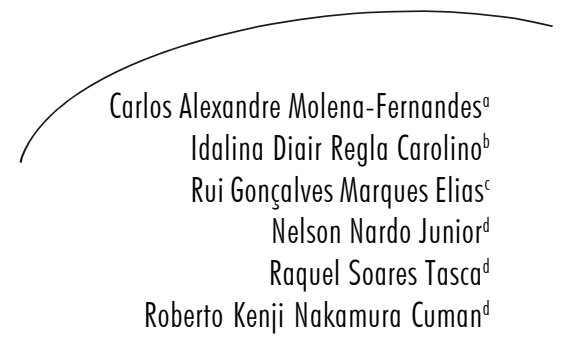

\section{Resumo}

Este estudo tem por objetivo verificar a influência de um programa de exercícios físicos aeróbios sobre o perfil lipídico de pacientes idosas, portadoras de Diabetes Mellitus (DM) tipo 2. Foram selecionados 40 pacientes do gênero feminino com idade maior ou igual a 60 anos. A amostra foi distribuída em dois grupos de 20 pacientes cada: Grupo Treinado, que recebeu orientação nutricional e participou das sessões supervisionadas de exercício físico aeróbio, e Grupo Controle, que recebeu somente orientação nutricional. A intervenção teve a duração de 12 semanas. $\mathrm{O}$ protocolo de exercício foi aplicado três vezes por semana, tendo cada sessão $1 \mathrm{~h}$ de duração. Os dados obtidos mostraram que o protocolo desenvolvido promoveu redução significativa nos triglicerídeos séricos (pré-teste $=190 \pm 76,67$ e pós-teste $=125,33$ $\pm 45,82 \mathrm{mg} / \mathrm{dL}, \mathrm{p}<0,05)$ e no LDL-colesterol (pré-teste $=147,98 \pm 29,98 \mathrm{e}$ pós-teste $=122,24 \pm 17,61 \mathrm{mg} / \mathrm{dL}, \mathrm{p}<0,05)$ no Grupo Treinado. Os resultados permitiram concluir que o programa de exercícios adotado promoveu melhora no perfil lipídico de pacientes idosas portadoras de DM tipo 2, demonstrando a importância da prática de atividade física orientada em Unidades Básicas de Saúde.

\section{Abstract}

This study aims to evaluate the influence of a physical exercise program on the lipid profile in type 2 diabetic elderly women. The patients were selected from a Basic Unit of Health (Mandacaru - NIS II, Maringá, Paraná), that

\author{
Palavras-chave: \\ atividade motora; \\ exercício; perfil de \\ saúde; \\ antropometria; \\ gorduras na dieta; \\ mulheres; saúde do \\ idoso; Diabetes \\ Mellitus tipo 2; \\ centros de saúde
}


attends 200 type 2 diabetic subjects. Among these patients, 40 women aged 60 years or more were randomically selected. The sample was distributed in two groups of 20 patients each: the Trained Group (TG), that received nutritional instructions and participated in supervised sessions of aerobic physical exercise; Control Group (CG), that received only nutritional instructions. The exercises were applied three times a week, one hour each session. Results showed that the adopted protocol promoted a significant reduction in triglycerides levels (pre-test $=190 \pm 76,67$ and post-test $=$ $125,33 \pm 45,82 \mathrm{mg} / \mathrm{dL}, \mathrm{p}<0,05)$ and also in the LDL-cholesterol (pre-test $=$ $147,98 \pm 29,98$ and post-test $=122,24 \pm 17,61 \mathrm{mg} / \mathrm{dL}, \mathrm{p}<0,05)$ for the TG. Therefore, we concluded that the adopted exercise program improved the lipid profile in type 2 diabetic elderly women, showing the importance of practicing oriented physical activity in health primary units.
Key-words: motor activity; exercise; health profile; anthropometry; dietary fats; women; health of the elderly; Diabetes Mellitus, type 2; health centers
INTRODUÇÃO

O processo de envelhecimento populacional no Brasil é um dos mais acelerados do mundo. Segundo dados do Instituto Brasileiro de Geografia e Estatística, ${ }^{1}$ referentes ao censo de 2000, do total de habitantes, 15,5 milhões tinham 60 anos ou mais, representando $10 \%$ da população geral. Projeções para 2025 indicam que esse número poderá ser superior a 30 milhões, correspondendo a $15 \%$ da população total estimada e colocando nosso país em sexto lugar do mundo no que se refere à população idosa em termos absolutos. ${ }^{2}$

Por outro lado, com o aumento progressivo da idade, há diminuição do envolvimento do número de pessoas em atividades físicas vigorosas, moderadas e da vida diária, que promovem decréscimo da capacidade física. ${ }^{3,4}$ Tal fato tem sido associado ao aumento da prevalência de doenças crônico-degenerativas não-transmissíveis, tais como: a doença coronariana, o aciden- te vascular cerebral, a osteoartrite, os problemas respiratórios, os desvios posturais, a obesidade, a hipertensão e o Diabetes Mellitus (DM) tipo 2..$^{5,6}$

O DM é uma das principais síndromes de evolução crônica que acometem a população nos dias atuais. Entre as diferentes classificações do diabetes, o DM tipo 2 é o de maior incidência, responsável por aproximadamente $90 \%$ dos casos. ${ }^{7,8}$ E evidente a epidemia de DM na atualidade, com projeções alarmantes para este século, especialmente nos países em desenvolvimento. ${ }^{9} \mathrm{Em}$ 1985 , eram 30 milhões de pacientes no mundo todo. Uma década mais tarde, o número quadruplicou (135 milhões). De acordo com a Organização Mundial da Saúde, ${ }^{10} \mathrm{em}$ 2000 existiam 177 milhões, mas esse quadro deverá aumentar para 370 milhões até 2030. Nas Américas, o número de indivíduos com diabetes foi estimado em $35 \mathrm{mi}$ lhões para o ano 2000 e projetado para 64 milhões em 2025. ${ }^{7}$ No Brasil, um estudo multicêntrico de base populacional, conduzido em 1988 em nove capitais, demonstrou 
prevalência de 7,6\% de diabetes na população urbana com idade de 30 a 69 anos. ${ }^{11}$

A idade do aparecimento do DM tipo 2 é variável, embora seja mais frequente após os 40 anos de idade, sendo a maior incidência ao redor dos 60 anos. ${ }^{12,13} \mathrm{Com}$ relação ao gênero, a incidência e a prevalência do DM tipo 2 é 1,4 a 1,8 vezes mais freqüente nas mulheres do que nos homens. ${ }^{14}$ Associando estes dados ao aumento da prevalência dessa enfermidade na população, estima-se que a maioria dos diabéticos, nos próximos anos, será constituída de mulheres idosas. ${ }^{8}$

Dados da OMS, ${ }^{10}$ mostram significativa elevação da mortalidade de indivíduos com DM, tipos 1 e 2, na presença de dislipidemias. Por outro lado, são numerosas as evidências de que o tratamento da dislipidemia tem se mostrado benéfico no controle de doenças vasculares em indivíduos diabéticos. ${ }^{15,16,17,18}$

O exercício físico regular promove melhora do perfil lipídico; sendo o exercício aeróbio aquele que mais atua no metabolismo das lipoproteínas que elevam a concentração sanguínea do HDL-colesterol e reduz o colesterol total. ${ }^{19}$ Além disso, reduz as concentrações plasmáticas dos triglicerídeos, apesar de a diminuição do LDL-colesterol ainda permanecer controversa. ${ }^{20,18}$

Sendo assim, o controle dos lipídeos sangüíneos, além da hiperglicemia, tem sido recomendado para pacientes diabéticos, já que as dislipidemias são as maiores responsá- veis pela morbidade e mortalidade dos portadores dessa patologia. ${ }^{21,22}$ Além disso, a American Diabetes Association ${ }^{23}$ refere que o tratamento inicial da dislipidemia em pacientes diabéticos deve ser o controle nutricional e a prática de atividade física.

Apesar de vários estudos recomendarem que o exercício físico deva fazer parte da terapia não-medicamentosa do DM tipo 2 há poucos relatos sobre o papel do exercício físico nas alterações lipídicas em pacientes idosos, portadores de DM tipo 2. Diante disso, o objetivo do presente estudo foi verificar a influência de um programa de exercícios físicos aeróbios sobre o perfil lipídico em pacientes idosas, portadoras de DM tipo 2.

\section{CASUÍSTICA E MÉTODOS}

As mulheres idosas selecionadas para compor a amostra deste estudo estavam cadastradas na Unidade Básica de Saúde Mandacaru - NIS II, Maringá, Estado do Paraná, que atende um total de 200 pessoas portadores de DM tipo 2. Os critérios de inclusão foram: a) ser portador de diabetes tipo 2 há pelo menos cinco anos e não apresentar nenhuma doença incapacitante para a prática de exercícios físicos; b) ser do sexo feminino, com idade maior ou igual a 60 anos; c) disponibilidade para participar das sessões de atividade física três vezes por semana e das avaliações bioquímicas e antropométricas; e d) não estar praticando atividade física regular há pelo menos seis me- 
ses. Assim, das 200 pessoas com DM tipo 2 cadastradas nessa Unidade Básica de Saúde, 40 foram selecionadas para este estudo, pois atenderam aos critérios acima descritos.

No início da pesquisa, todas as mulheres selecionadas foram esclarecidas quanto aos objetivos e à metodologia a ser utiliza$\mathrm{da}$, tendo sido obtido o consentimento individual por escrito. $O$ presente trabalho teve aprovação do Comitê de Ética em Humanos da Universidade Estadual de Maringá (Registro n 092/2004. Parecer n 178/ 2004). Num segundo momento, as $40 \mathrm{mu}-$ lheres selecionadas foram distribuídas de maneira aleatória, por meio de sorteio, formando dois grupos de 20, conforme descrição a seguir: Grupo Treinado $(\mathrm{T})$ - recebeu orientação nutricional e participou das sessões supervisionadas de exercício físico aeróbio; Grupo Controle (C) - recebeu somente orientação nutricional.

A intervenção teve a duração de 12 semanas, no período de agosto a outubro de 2005. O protocolo de exercício foi aplicado três vezes por semana, em dias alternados, sendo uma sessão por dia e cada sessão com uma hora de duração.

\section{Protocolo das Sessões de Exercício Físico}

a) Fase inicial (8 min.) - exercícios para aquecimento articular e fisiológico;

b) Fase principal (45 min. de exercício aeróbio) - caminhada acelerada, mantendo intensidade de leve a moderada (frequência cardíaca (FC) entre 50\% a 80\% da FC máxima para a idade), segundo o American College of Sports Medicine. ${ }^{24}$

c) Fase final (7 min.) - exercícios de alongamento muscular e relaxamento, visando ao retorno da FC aos níveis de repouso e melhoria da flexibilidade.

\section{Instrumentos}

Foi aplicado um questionário estruturado abordando: a) características demográficas e socioeconômicas das pacientes (idade, estado civil, nível de escolaridade, número de pessoas no domicílio e renda familiar mensal); b)características do padrão de atividade física (frequiência e duração da prática de exercícios físicos); c) características clínicas (antecedentes patológicos familiares e história clínica pessoal); d) freqüência do consumo de cigarros e de bebidas alcoólicas; e e) uso de medicamentos.

Os questionários foram aplicados em forma de entrevista por uma equipe previamente treinada, composta por entrevistadores com nível de escolaridade superior e com experiência prévia de trabalho de campo. A coleta de dados foi realizada antes e após o período de intervenção. As medidas antropométricas (peso, altura e circunferências da cintura e quadril) foram realizadas em duplicata, sendo utilizada a média aritmética dos valores.

Para as medidas de estatura, foi utilizado um estadiômetro de madeira, com escala de precisão de $0,1 \mathrm{~cm}$ com um cursor 
acoplado para facilitar a leitura. A determinação da estatura foi realizada pela colocação da avaliada descalça posicionada sobre a base do estadiômetro, postura ereta, com os pés unidos, membros superiores pendentes ao longo do corpo, tocando as superfícies posteriores do corpo na escala de medidas. Utilizando-se o cursor, foi determinada a medida correspondente à distância entre a região plantar e o vértex, estando a avaliada em apnéia inspiratória e com a cabeça orientada no plano de Frankfurt paralela ao solo.

As medidas de peso corporal foram realizadas, e foi utilizada uma balança de alavanca, marca Filizola, com precisão de 100 g. No momento da realização dessa medida, a avaliada estava descalça e com o mínimo de roupa possível. Para o registro da medida, solicitou-se que a avaliada se posicionasse no centro da plataforma da balança e se mantivesse ereta, com os braços ao longo do corpo e com o olhar fixo à sua frente, para que não ocorressem oscilações no momento do registro da medida. A balança foi aferida antes do início das pesagens e a cada dez pesagens. A partir dos valores obtidos com as medidas de estatura e peso corporal, foi calculado o índice de Quetelet ou índice de massa corpórea (IMC), de acordo com a seguinte relação: $\mathrm{IMC}=$ Peso corporal $(\mathrm{kg}) \div$ Estatura $^{2}(\mathrm{~m})$.

A cintura foi medida em $\mathrm{cm}$, com fita métrica inelástica, no ponto médio entre a crista ilíaca e a face externa da última costela e o quadril também em centímetros, com fita inelástica, foi medido no ponto onde se localiza o perímetro de maior extensão entre os quadris e as nádegas. A relação cintura-quadril (RCQ) foi obtida pelo quociente entre as circunferências da cintura e do quadril. Os dados referentes ao perfil lipídico e glicemia foram obtidos dos prontuários dos pacientes fornecidos pela Unidade Básica de Saúde Mandacaru - NIS II.

\section{Análise Estatística}

A análise estatística foi realizada por meio do Programa Epi-Info (versão 6.0). Os resultados foram expressos em frequiência e percentual para as variáveis qualitativas e em média \pm desvio-padrão para as variáveis quantitativas. As diferenças pré e pós-teste foram determinadas por meio do teste ' $t$ ' de Student, e $\mathrm{P}<0,05$ foi utilizado como nível de significância. Utilizou-se, ainda, o delta percentual $(\Delta \%)$ para verificar as diferenças pré e pós-intervenção em cada grupo em termos percentuais.

\section{RESULTADOS}

Das 20 mulheres selecionadas para o Grupo Treinado, todas aderiram às sessões de exercício na primeira semana. Entretanto, ao longo do estudo ocorreu uma evasão de 40\% (8 pacientes). Entre as 12 pacientes restantes deste grupo, a freqüência média de participação nas sessões de atividade física foi de $92 \%$. Destas, uma não realizou as avaliações antropométricas finais. Diante disto, ao final dos três meses de intervenção, o Grupo Treinado 
apresentou índice de desistência de $45 \%$. Sendo assim, considerou-se para este estudo uma população de 11 pacientes que participaram efetivamente das caminhadas e realizaram todos os testes.

Em relação ao Grupo Controle, que também iniciou o estudo com 20 pacientes, a perda amostral foi menor. Ao final do período de intervenção, apenas quatro pacientes (20\%) não realizaram os exames e as avaliações antropométricas, ficando a amostra final constituída de 16 pacientes.
Na tabela 1 , verifica-se freqüência maior de pacientes na faixa etária de 60 a 69 anos (74\%). Em relação à escolaridade, observouse baixo nível de instrução entre as pacientes, sendo que $30 \%$ eram analfabetas, $63 \%$ cursaram o ensino fundamental e apenas $7 \%$ concluíram o ensino médio. Os dados de renda per capita indicaram baixo nível econômico da população, com $74 \%$ das pacientes recebendo até um salário mínimo; $22 \%$ recebendo de um a três salários e apenas $4 \%$ recebendo acima de três salários mínimos percapita mensalmente.

Tabela 1 - Características sóciodemográficas das pacientes idosas com DM tipo 2 atendidas na Unidade Básica de Saúde NIS - II Mandacaru, Maringá - Estado do Paraná, 2004.

\begin{tabular}{|c|c|c|}
\hline Variáveis & Frequência & $\%$ \\
\hline \multicolumn{3}{|l|}{ Idade (anos) } \\
\hline $60-69$ & 20 & 74 \\
\hline $70-79$ & 06 & 22 \\
\hline 80 anos ou mais & 01 & 04 \\
\hline \multicolumn{3}{|l|}{ Escolaridade } \\
\hline Nenhuma & 08 & 30 \\
\hline $1^{\mathrm{a}}$ a $4^{\mathrm{a}}$ séries (Ensino Fundamental) & 10 & 37 \\
\hline $5^{a}$ a $8^{a}$ séries (Ensino Fundamental) & 07 & 26 \\
\hline Ensino Médio & 02 & 07 \\
\hline \multicolumn{3}{|l|}{$\begin{array}{l}\text { Renda Familiar per capita } \\
\text { (salário-mínimo) }\end{array}$} \\
\hline$<0,5$ & 03 & 11 \\
\hline $0,5-0,99$ & 17 & 63 \\
\hline $1-1,99$ & 04 & 15 \\
\hline $2-2,99$ & 02 & 07 \\
\hline 3 ou mais & 01 & 04 \\
\hline
\end{tabular}

A média de idade entre os grupos estudados foi semelhante, sendo de 68,13 anos (Grupo Controle) e 68,55 (Grupo Treinado). Comparando-se os parâmetros antropométricos e bioquímicos iniciais, observou-se também distribuição bastante homogênea entre os grupos, não sendo observadas diferenças estatisticamente significativas em nenhuma variável (tabela2). 
Importante destacar que os valores médios de IMC, circunferência da cintura e RCQ indicam uma população de pacientes acima do peso, com obesidade abdominal (visceral) predominante. Os dados obtidos no Grupo Controle indicam um IMC médio de $29,9 \mathrm{~kg} / \mathrm{m}^{2}$, limítrofe para obesidade, e no Grupo Treinado a média do IMC foi de $30,18 \mathrm{~kg} / \mathrm{m}^{2}$, valor este classificado como obesidade. ${ }^{25}$ Em relação à medida da circunferência da cintura, os dois grupos apresentaram uma média de $103 \mathrm{~cm}$, valor muito superior aos $88 \mathrm{~cm}$ considerado como ponto de corte para indicar obesidade visceral (andróide) em mulheres. ${ }^{26}$ Os valores da RCQ também confirmam a presença de obesidade visceral nos grupos estudados, com valores de 0,98 e 0,99, nos Grupos Controle e Treinado, respectivamente. Estes valores também estão bem acima dos va- lores de corte estabelecidos como indicadores de obesidade andróide, 0,85 para mulheres. $^{27}$

Em relação às variáveis antropométricas, em ambos os grupos não se observaram diferenças significativas em nenhuma variável após os três meses de intervenção (tabela 2). Entretanto, foi verificada discreta diminuição de $1,31 \mathrm{~kg}(-1,8 \%)$ na média do peso corporal do Grupo Treinado, enquanto que no Grupo Controle esta redução foi de $0,45 \mathrm{~kg}$ $(-0,62 \%)$. Conseqüentemente, houve pequena redução no IMC nos grupos estudados e, no Grupo Treinado, o IMC passou de 30,18 $\mathrm{Kg} / \mathrm{m}^{2}$ (faixa de obesidade) para $29,63 \mathrm{Kg} /$ $\mathrm{m}^{2}$ (faixa de sobrepeso), após a intervenção. Neste mesmo grupo, em relação à circunferência da cintura, foi observada diminuição média de $1 \mathrm{~cm}(-1 \%)$ com o treinamento.

Tabela 2 - Características antropométricas dos pacientes Diabéticos Exercitados (Grupo Treinado) e Diabéticos não exercitados (Grupo Controle) pré (antes) e pós (após) a intervenção. Maringá, PR, 2004.

\begin{tabular}{|c|c|c|c|c|c|c|}
\hline & \multicolumn{3}{|c|}{ Grupo Controle $(n=16)$} & \multicolumn{3}{|c|}{ Grupo Treinado $(n=11)$} \\
\hline & Pré & Pós & $\Delta \%$ & Pré & Pós & $\Delta \%$ \\
\hline PESO (Kg) & $72,6 \pm 12,38$ & $72,15 \pm 11,52$ & $-0,62$ & $70,6 \pm 10,1$ & $69,29 \pm 9,62$ & $-1,9$ \\
\hline ESTATURA (m) & $1,56 \pm 0,06$ & $1,56 \pm 0,06$ & 0,0 & $1,53 \pm 0,07$ & $1,53 \pm 0,07$ & 0,0 \\
\hline $\operatorname{IMC}\left(\mathrm{Kg} / \mathrm{m}^{2}\right)$ & $29,91 \pm 4,56$ & $29,75 \pm 4,31$ & $-0,55$ & $30,18 \pm 3,78$ & $29,63 \pm 3,73$ & $-1,8$ \\
\hline CINTURA (cm) & $103,25 \pm 10,10$ & $103,31 \pm 10,03$ & 0,06 & $103,4 \pm 10,10$ & $102,4 \pm 10,15$ & -1 \\
\hline QUADRIL (cm) & $105,63 \pm 12,54$ & $105,67 \pm 12,43$ & 0,04 & $104,3 \pm 10,89$ & $104 \pm 10,45$ & $-0,3$ \\
\hline RCQ & $0,98 \pm 0,08$ & $0,98 \pm 0,09$ & 0,0 & $0,99 \pm 0,07$ & $0,98 \pm 0,07$ & -1 \\
\hline
\end{tabular}

Observou-se, conforme a tabela 3, que nenhum parâmetro bioquímico sofreu alteração significativa no Grupo Controle após o período de intervenção. Quanto ao Grupo Treinado, após o programa de três meses de exercício físico aeróbio, observou-se diminuição estatisticamente significativa nos valores médios do LDL-co- 
lesterol e dos triglicerídeos. Em relação às outras variáveis bioquímicas, apesar de não apresentarem diferenças significativas, houve importante decréscimo nas médias da glicemia $(14,81 \%)$, do colesterol total $(9,24 \%)$ e do VLDL-colesterol $(34,19 \%)$, além de considerável aumento no HDLcolesterol (12,18\%).

Tabela 3 - Características bioquímicas dos pacientes Diabéticos Exercitados (Grupo Treinado) e Diabéticos não exercitados (Grupo Controle) pré (antes) e pós (após) a intervenção. Maringá, PR, 2004.

\begin{tabular}{lcccccc}
\hline \multicolumn{1}{c}{$\begin{array}{c}\text { Concentrações em } \\
(\mathrm{mg} / \mathrm{dl})\end{array}$} & \multicolumn{2}{c}{ Grupo Controle $(\mathrm{n}=16)$} & \multicolumn{3}{c}{ Grupo Treinado $(\mathrm{n}=11)$} \\
\cline { 2 - 7 } & \multicolumn{1}{c}{ Pré } & \multicolumn{1}{c}{ Pós } & $\Delta \%$ & Pré & Pós & $\Delta \%$ \\
\hline GLICEMIA & $139,3 \pm 34,4$ & $140,5 \pm 36,8$ & 0,8 & $158,8 \pm 60,0$ & $135,3 \pm 45,4$ & $-14,8$ \\
COLESTEROL TOTAL & $218,5 \pm 39,2$ & $206,5 \pm 32,1$ & $-5,5$ & $222,6 \pm 31,7$ & $202,0 \pm 23,4$ & $-9,2$ \\
HDL-colesterol & $43,5 \pm 9,1$ & $45,7 \pm 16,8$ & 5,2 & $48,8 \pm 9,8$ & $54,8 \pm 13,7$ & 12,2 \\
LDL-colesterol & $139,3 \pm 28,7$ & $130,1 \pm 19,2$ & $-6,6$ & $147,9 \pm 29,9$ & $122,2 \pm 17,6^{*}$ & $-17,4$ \\
VLDL-colesterol & $35,8 \pm 13,2$ & $31,2 \pm 11,6$ & $-12,7$ & $38,0 \pm 15,3$ & $25,0 \pm 9,2$ & $-34,2$ \\
TRIGLICERÍdEOS & $178,9 \pm 66,2$ & $156,8 \pm 57,8$ & $-12,3$ & $190,3 \pm 76,7$ & $125,3 \pm 45,8^{*}$ & $-34,1$ \\
\hline
\end{tabular}

* Diferença significativa entre os momentos pré e pós-intervenção ( $\mathrm{p}<0,05, t$ de Student).

\section{DISCUSSÃO}

Neste estudo observou-se baixo índice de aderência ao programa de intervenção. Apesar das vantagens da prática regular de atividade física, grande parte dos diabéticos é inativa ou se exercita em níveis insuficientes para alcançar resultados satisfatórios para a saúde. ${ }^{28} \mathrm{~A}$ baixa adesão ao tratamento constitui um dos maiores problemas enfrentados pelos profissionais de saúde. A aderência é pior em situações que requerem tratamentos longos, de natureza preventiva e quando há necessidade de alteração no estilo de vida, como é o caso da atividade física. ${ }^{29}$

Dados da literatura têm apontado que a maioria das desistências ocorre durante os três meses iniciais, com resultados semelhantes em todas faixas etárias, independentemente do sexo. ${ }^{28,30} \mathrm{Uma}$ extensa revisão apontou que apenas $19 \%$ a 30\% dos pacientes diabéticos aderem às prescrições de exercícios. ${ }^{31} \mathrm{O}$ grau de aderência à atividade física, neste trabalho, foi de $55 \%$, valor superior aos dados encontrados em outros estudos, evidenciando que pessoas idosas tendem a aderir mais à prática regular de atividades físicas dos que os adultos em geral, fato também observado em outras pesquisas. ${ }^{32,33}$

Quanto aos dados sociodemográficos, a população estudada apresentava, em sua maioria (74\%), indivíduos compreendidos entre a faixa dos 60-69 anos de idade. Observa-se, ainda, que se trata de uma população de baixa ou nenhuma escolaridade e de 
baixo poder aquisitivo. Em estudo realizado na mesma Unidade Básica de Saúde, foram encontrados valores semelhantes em relação às variáveis socioeconômicas, ${ }^{34}$ mas para pacientes portadores de hipertensão, doença co-existente ao DM tipo 2.

$\mathrm{O}$ excesso de peso atinge cerca de um terço da população adulta e tem apresentado tendência crescente nas últimas décadas, mesmo entre as pessoas idosas. ${ }^{8}$ Estima-se que entre $80 \%$ e $90 \%$ dos indivíduos acometidos pelo DM tipo 2 são obesos ou estão acima do peso. ${ }^{35,36}$

Em nossa pesquisa observamos, pela avaliação antropométrica, que os pacientes apresentavam excesso de peso, com obesidade visceral predominante, confirmando dados de outros estudos que revelam que a obesidade e/ou sobrepeso estão presentes na maioria dos pacientes diabéticos tipo $2 .{ }^{22,18}$

O perfil bioquímico dos dois grupos (Controle e Treinado) indicou que as pacientes apresentavam alterações importantes no perfil lipídico. Essas alterações podem contribuir significativamente para aumentar o risco de doenças cardiovasculares em pacientes com diabetes. Os dois principais componentes da dislipidemia diabética são os níveis elevados de triglicerídeos e os baixos níveis de HDL-colesterol, ambos considerados os maiores preditores de doenças cardiovasculares. ${ }^{22,18}$

Neste estudo, os valores médios iniciais dos triglicerídeos foram de $178,89 \mathrm{mg} / \mathrm{dL}$ para o Grupo Controle e de 190,3 mg/dl para o Grupo Treinado, valores estes superiores a $150 \mathrm{mg} / \mathrm{dL}$, recomendado pela ADA. ${ }^{18}$ Quanto ao HDL-colesterol, a ADA ${ }^{18}$ recomenda que, para pacientes diabéticos, o valor de referência deve ser superior a $45 \mathrm{mg}$ / dL. Em nosso estudo, observamos que os valores médios do HDL-colesterol do Grupo Controle estavam reduzidos $(43,48 \mathrm{mg}$ / $\mathrm{dL}$ ), enquanto que no Grupo Treinado foram mais elevados $(48,82 \mathrm{mg} / \mathrm{dL})$.

Estudos têm verificado que por meio de mudanças no estilo de vida, como uma melhor dieta e incremento da atividade física, é possível melhorar o quadro de dislipidemia em diabéticos, principalmente na redução dos triglicerídeos e aumento do HDL-colesterol. ${ }^{8,18,37,38,39}$ Entretanto, em relação ao LDL-colesterol, os dados da literatura são menos conclusivos, mas a maioria dos pacientes diabéticos possui taxas elevadas de LDL-colesterol. De acordo com os resultados do Third National Health and Nutrition Examination Survey (NHANES III), ${ }^{40}$ por exemplo, $84,6 \%$ dos pacientes com DM tipo 2 têm os níveis de LDL-colesterol acima do ótimo $(100 \mathrm{mg} / \mathrm{dL})$.Neste estudo, também observamos valores médios de LDL-colesterol acima do recomendado (139,28 e 147,98 $\mathrm{mg} / \mathrm{dL}$ ) para os grupos Controle e Treinado, respectivamente. Nesse sentido, observou-se que a amostra selecionada para este estudo apresentava, em seu início, dois importantes fatores de risco cardiovascular associados ao DM tipo 2: obesidade e dislipidemia.

Em relação às variáveis antropométricas, não foram observadas diferenças significativas após a intervenção. Entretanto, verifi- 
cou-se diminuição discreta nos valores médios do peso $(-1,31 \mathrm{~kg})$, no IMC $(-0,55)$ na circunferência da cintura $(-1 \mathrm{~cm})$ e do quadril $(-0,3 \mathrm{~cm})$ e na RCQ $(-0,01)$ enquanto que no Grupo Controle, os valores médios se mantiveram semelhantes ou ate aumentaram em algumas variáveis. Estes dados corroboram achados de outros estudos que não encontraram diferenças significativas na massa corporal de sujeitos diabéticos submetidos ao exercício físico. ${ }^{21,41,42,43}$

Entretanto, outros estudos evidenciaram que o treinamento com exercícios físicos aeróbios diminui a massa corporal em indivíduos com diabetes. ${ }^{44,45,46} \mathrm{~A}$ maioria destes estudos, além da intervenção através da atividade física, associou uma dieta controlada aos pacientes.

Quanto à glicemia de jejum, apesar de não ser observada diferença significativa pós-intervenção, a diminuição em termos percentuais para o Grupo Treinado foi bastante superior ao Grupo Controle $(14,81 \%$ versus $0,83 \%$ ). Dados obtidos, após a realização de uma extensa meta-análise, revelaram que a prática regular de exercício físico reduz a hemoglobina glicosilada em pacientes com DM tipo 2. ${ }^{21}$ Entretanto, quando se trata da glicose sangüínea de jejum, alguns estudos evidenciam que o exercício físico reduz os níveis de glicemia em diabéti$\cos ,{ }^{42,44,45,46}$ enquanto que, em outros estudos, não se demonstrou esse benefício de forma conclusiva. ${ }^{47,48}$

Quanto aos lipídios sangüíneos, após a intervenção observamos diferenças signifi- cativas para os valores de triglicerídeos e de LDL-colesterol no Grupo Treinado. Embora não significativa, este mesmo Grupo apresentou valores médios de colesterol total e VLDL-colesterol menores, e o HDLcolesterol maior, após o programa de exercício, sugerindo que o exercício físico pode minimizar este risco cardiovascular em $\mathrm{pa}$ cientes com DM tipo 2. No Grupo Controle, não foi observada diferença significativa em nenhuma variável bioquímica, apesar de uma ligeira diminuição no colesterol total, LDL-colesterol e triglicerídeos, supostamente causada pela provável mudança nos hábitos alimentares pela orientação nutricional recebida por este grupo durante o período de intervenção.

Diversos estudos têm destacado o importante papel do exercício físico na diminuição dos triglicerídeos e no aumento do HDL-colesterol em pacientes portadores do DM tipo 2. ${ }^{18,39,46}$ Entretanto, poucos estudos demonstram efeito significativo na melhora dos níveis de LDL-colesterol em sujeitos portadores de DM, tipo $2,{ }^{18}$ fato este divergente dos resultados observados neste estudo, no qual os valores do LDLcolesterol se reduziram significativamente e os valores de HDL-colesterol aumentaram modestamente.

Os resultados deste estudo mostraram um papel benéfico do exercício em melhorar o perfil lipídico das pacientes idosas com DM tipo 2. Observou-se redução significativa nos triglicerídeos e no LDL-colesterol. Além disso, os níveis médios de HDL-colesterol aumentaram 
em 12,18\% no Grupo Treinado, contra 5,15\% do Grupo Controle. Estes dados corroboram os resultados de outros estudos que também demonstraram que a resposta ao exercício físico melhora os níveis de colesterol total, HDL-colesterol, LDLcolesterol e triglicerídeos. ${ }^{44,45}$ Segundo a $\mathrm{ADA},{ }^{18}$ a atividade física tem demonstrado ser eficiente também em diminuir o nível de VLDL-colesterol em indivíduos com DM tipo 2. No presente estudo, apesar de não significativa, observou-se redução nos valores médios de VLDL do Grupo Treinado de $34,19 \%$, após o programa de exercício físico, enquanto no Grupo Controle esta redução foi de $11,63 \%$.

Considerando os critérios de um bom controle metabólico para o paciente diabético tipo $2,{ }^{22}$ em relação à glicemia de jejum, o valor médio após a intervenção $(135,28 \mathrm{mg} / \mathrm{dL})$ estava dentro dos níveis aceitáveis $(116-140 \mathrm{mg} / \mathrm{dL})$. Quanto ao HDLcolesterol, o valor médio final de $54,77 \mathrm{mg} /$ dL também foi considerado como bom para mulheres diabéticas $(>45)$. Após a intervenção, os valores sangüíneos de triglicerídeos e do LDL-colesterol apresentaram valores significativamente melhores, enquadrandose inclusive na faixa da normalidade para pacientes com DM, tipo 2, demonstrando o papel do exercício físico na normalização destes parâmetros bioquímicos.

\section{CONCLUSÕES}

O programa regular de exercício físico aplicado no presente estudo melhorou os níveis de lipídios sangüíneos na população estudada, diminuindo significativamente os valores médios dos triglicerídeos e do LDLcolesterol, e aumentando consideravelmente os valores de HDL-colesterol.

Não ficou evidente, neste estudo, o efeito do exercício físico na redução da massa corporal e da glicemia de jejum das pacientes com DM tipo 2, apesar de ter sido observada discreta redução no peso corporal, IMC e nos valores médios da glicose sangüínea no Grupo Treinado.

Os resultados deste estudo permitiram concluir que um programa regular de exercícios físicos aeróbios, de intensidade moderada, promoveu melhoria no perfil lipídico em idosas portadoras de DM tipo 2, uma vez que o Grupo Treinado, após o programa de três meses de exercício físico aeróbio, apresentou diminuição estatisticamente significativa nos valores médios do LDL-colesterol e dos triglicerídeos, além de considerável aumento no HDL-colesterol.

\section{NOTAS}

\footnotetext{
Professor de Educação Física, Doutorando em Ciências Farmacêuticas, Universidade Estadual de Maringá, Docente da Faculdade Estadual de Educação, Ciências e Letras de Paranavaí (FAFIPA).

b Nutricionista, Mestra, Docente da Universidade Estadual de Maringá, Paraná.

Professor de Educação Física, Mestre em Ciências da Saúde, Universidade Estadual de Maringá.

d Professores Doutores, Universidade Estadual de Maringá, Paraná.
} 


\section{REFERÊNCIAS}

1. IBGE. Censo demográfico 2000. Características da população e dos domicílios resultados do universo. Brasília: IBGE; 2000.

2. Organización Panamericana de la Salud. Envejecimiento y salud: un cambio de paradigma. Rev Panam Salud Publica 2000; 7: 60-7.

3. Sallis JF. Age-related decline in physical activity: a synthesis of human and animal studies. Med Sci Sports Exerc 2000; 32:1598-600.

4. Matsudo SMM. Atividade física e envelhecimento: aspectos epidemiológicos. In: Matsudo SMM, editor. Envelhecimento e atividade física. 1. ed. Londrina: Midiograf; 2001. p.60-70.

5. Rockhill B, Willet WC, Manson JE, Leitzmann MF, Stampfer MJ, Hunter DJ, et al. Physical activity and mortality: a prospective study among women. Am J Public Health 2001; 91:578-83.

6. Sesso HD, Paffenbarger RS, Ha T, Lee IM. Physical activity and cardiovascular disease risk in middle-age and older women. Am J Epidemiol 1999;150: 408-16.

7. Barceló A, Rajpathak S. Incidence and prevalence of diabetes mellitus in the Americas. Rev Panam Salud Publica 2001; 10: 300-08.

8. Organização Pan-Americana da Saúde. Doenças crônico-degenerativas: estratégia mundial sobre alimentação saudável, atividade física e saúde. Brasília: Organização Pan-Americana da Saúde; 2003.60p.

9. Ferreira M, Matsudo S, Matsudo V, Braggion, G. Efeitos de um programa de orientação de atividade física e nutricional sobre o nível de atividade física de mulheres fisicamente ativas de 50 a 72 anos de idade. Revista brasileira de medicina do esporte 2005; 11: 172-76.

10. World Health Organization. The World Health Report 2003. Geneva: World Health Organization; 2003.

11. Malerbi DA, Franco LJ. Multicenter study of the prevalence of diabetes mellitus and impared glucose tolerance in the urban Brazilian population aged 30-69 years. Diabetes Care 1992; 15(11): 1509-16.

12. Gross JL, Silveiro SP, Camargo JL, Reichelt AJ, Azevedo MJ. Diabetes melito: diagnóstico, classificação e avaliação do controle glicêmico. Arq Bras Endocrinol Metabol 2002; 46: 16-26.

13. Goldenberg P, Schenkman, S, Franco LJ. Prevalência de diabetes mellitus: diferenças de gênero e igualdade entre os sexos. Revista brasileira de epidemiologia 2003; 6: 18-28.

14. Brasil. Ministério da Saúde.Cadernos de Atenção Básica: Hipertensão arterial sistêmica - HAS e Diabetes mellitus - DM Protocolo. Brasil: 2001.

15. Hafner MP, Stern HP, Hazuda BD, Mitchell JK, Patterson E. Parental hitory of diabetes is association with increased cardiovascular risk factors. Arteriosclerosis 1989; 9: 928-33.

16. Heart Protection Study Group. MRC/ BHF Heart Protection Study of antioxidant vitamin supplementation in 50.5366 high-risk individuals: a randomized placebo-controlled trial. The Lancet 2002; 360: 23-33.

17. Khawali C, Andriolo A, Ferreira SRG. Benefícios da atividade física no perfil lipídico de pacientes com diabetes tipo 1. Arq Bras Endocrinol Metab 2003; 47 (1): 49-54. 
18. American Diabetes Association Standards of Medical Care in Diabetes. American Diabetes Association: Clinical Practice Recommendations 2004: Position Statement. Diabetes Care 2004; 27( suppl 1):15-35.

19. Couillard C, Despres JP, Lamarche B, Bergeron J, Gagnon J. Efects of endurance exercise training on plasma $\mathrm{HDL}$ cholesterol levels depend on levels og triglycerides: evidence from men of the health risk factors, exercise training and genetics (HERITAGE) family study. Arterioscler Thromb Vasc Biol 2001; 21 (7): 1226-32.

20. Thomas TR, La Fontaine T. Exercise and lipoproteins. In: American College of Sports Medicine. ACSM's resource manual for guidelines for exercise testing and prescription. $3^{\text {rd }}$ ed. Baltimore : Williams \& Wilkins; 1998. p. 294-301

21. Boulé NG, Haddad E, Kenny GP, Wells GA, Sigal RJ. Effects of exercise on glycemic control and body mass in type 2 diabetes mellitus. A meta-analysis of controlled clinical trials. JAMA 2001; 286: 1218-27.

22. Sociedade Brasileira de Diabetes. Consenso brasileiro sobre diabetes 2002: diagnóstico e classificação do diabetes melito e tratamento do diabetes melito do tipo 2. Rio de Janeiro: Diagraphic; 2003.

23. American Diabetes Association. Dyslipidemia management in adults with diabetes. Diabetes Care 2004; 27(1): 68-71.

24. American College of Sports Medicine. Research manual for guidelines for exercise testing and prevention. 2nd ed. Philadelphia: Lea and Febiger, 1993.

25. World Health Organization. Preventing and managing the global epidemic of obesity. [Report of the World Health Organization Consultation of Obesity]. Geneva; 1997.
26. Lean MEJ, Han TS, Morrison CE. Waist circumference as a measure for indicating need for weight management. BMJ 1995; 311: 158-61.

27. Avery CS. Abdominal obesity: scaling down this deadly risk. Phys Sportsmed 1991; 9(10): 137.

28. Fechio JJ, Malerbi FEK. Adesão a um programa de atividade física em adultos portadores de diabetes. Arq Bras Endocrinol Metabol 2003; 48(2):267-75.

29. Dunbar-Jacob J, Burka LE, Puczynski S. Clinical assessment and management of adherence to medical regimens. In: Nicassio PM, Smith TW, editors. Managing chronic illness: a biopsychosocial perspective. Washington: American Psychological Association; 1996. p.313-49.

30. Martin JE, Dubbert PM. Exercise applications and promotion in Behavioral Medicine: current status and future directions. J Consult Clin Psychol 1982; 50: 1004-17.

31. Lutfey KE, Wishner WJ. Beyond "compliance" is "adherence". Diabetes Care 1999; 22: 635-9.

32. Guillet PA, White AT, Caserta MS. Effects of exercise and/or fitness education on fitness in older, sedentary, obese women. J Aging Phys Act 1996; 4: 42-55.

33. Mayer JA, Jermanovich A, Wright BL, Elder JP, Drew JA, Williams SJ. Changes in health behaviors of older adults: The San Diego Medicare Preventive Health Project. Prev Med 1999; 23: 127-33.

34. Tasca RS, Soares DA, Cuman RKN. Acesso a medicamentos anti-hipertensivos em unidade básica de saúde em MaringáParaná. Arquivos de ciencias da sauide da UNIPAR 1999; 3(2): 117-23. 
35. King H, Kriska AM. Prevention of type II Diabetes by physical training. Epidemiological considerations and study methods. Diabetes Care 1998; 15 (11): 1794-9.

36. Sartorelli DS, Franco LJ. Tendências do diabetes mellitus no Brasil: o papel da transição nutricional. Cad Saúde Pública 2003; 19 (Sup 1): 29-36.

37. Lehto S, Ronnemaa T, Haffner SM, Pyörälä K, Kallio V, Laakso M.

Dyslipidemia and hyperglycemia predict coronary heart disease events in middleaged patients with NIDDM. Diabetes 1997; 46:1354-59.

38. Hamman RF. Prevention of type 2 Diabetes. In: Williams R, Herman W, Kinmonth AL, Wareham NJ, editors. Evidence Base for Diabetes Care. Chichester; Hoboken, N.J. : J. Wiley \& Sons, 2002

39. Taskinen MR. Controlling lipid levels in diabetes. Acta Diabetol 2002; 39: 29-34.

40. National Center for Health Statistic. Centers for Disease Control and Prevention. National health and nutrition examination survey (NHANES III). Atlanta: National Heart, Lung and Blood Institute; 1991.

41. Vanninen E, Uusitupa M, Siltonen O, Laitinen J, Lansimies E. Habitual physical activity, aerobic capacity and metabolic control in patients with newlydiagnosed type 2 diabetes mellitus: effect of 1-year diet and exercise intervention. Diabetologia 1992; 35: 340-6.

42. Raz I, Hauser E, Burszlyn M. Moderate exercise improves glucose metabolism in uncontrolled elderly patients with noninsulin-dependent diabetes mellitus. Isr J Med Sci 1994; 30: 766-70.

43. Honkola A, Forsen T, Erickson J. Resistance training improves the metabolic profile in individuals with type 2 diabetes. Acta Diabetol 1997; 34: 245-8.

44. Leong KS, Wilding JP. Obesity and diabetes. Baillieres Clin Endocrinol Metab 1999;13(2):221-37.

45. Kannan V. Diet, activity and diabetes. J Assoc Physicians India 1999;47(8):761-3.

46. Silva CA, Lima WC. Efeito benéfico do exercício físico no controle metabólico do diabetes mellitus tipo 2 a curto prazo. Arq Bras Endocrinol Metabol 2002; 46(5): 550-6.

47. Abe R, Fujinuma H. Exercise in elderly NIDDM. Nippon Ronen Igakkai Zasshi 1993; 30 (4):283-7.

48. Blonck MC, Jacobs MA, Biesheuvel EH, Weeda-Mannak WL, Heine RJ. Influences on weight loss in type 2 diabetic patients: little long-term benefit from group behaviour therapy and exercise training. Diabet Med 1994;11(5):449-57. 